\title{
Multimodal approach in assessment of the response of breast cancer to neoadjuvant chemotherapy
}

\author{
Mikhail Viktorovich Pavlov \\ Tatyana Igorevna Kalganova \\ Yekaterina Sergeevna Lyubimtseva \\ Vladimir Ivanovich Plekhanov \\ German Yurievich Golubyatnikov \\ Olga Yevgenyevna Ilyinskaya \\ Anna Gennadjevna Orlova \\ Pavel Vladimirovich Subochev \\ Dmitriy Vladimirovich Safonov \\ Natalia Mikhailovna Shakhova \\ Anna Vladimirovna Maslennikova
}

Mikhail Viktorovich Pavlov, Tatyana Igorevna Kalganova, Yekaterina Sergeevna Lyubimtseva, Vladimir Ivanovich Plekhanov, German Yurievich Golubyatnikov, Olga Yevgenyevna Ilyinskaya, Anna Gennadjevna Orlova, Pavel Vladimirovich Subochev, Dmitriy Vladimirovich Safonov, Natalia Mikhailovna Shakhova, Anna Vladimirovna Maslennikova, "Multimodal approach in assessment of the 


\title{
Multimodal approach in assessment of the response of breast cancer to neoadjuvant chemotherapy
}

\author{
Mikhail Viktorovich Pavlov, ${ }^{\mathrm{a}, \boldsymbol{}}$ Tatyana Igorevna Kalganova, ${ }^{\mathrm{b}}$ Yekaterina Sergeevna Lyubimtseva, ${ }^{\mathrm{c}}$ \\ Vladimir Ivanovich Plekhanov ${ }^{\mathrm{d}}$ German Yurievich Golubyatnikov, ${ }^{\mathrm{d}}$ Olga Yevgenyevna llyinskaya, ${ }^{\mathrm{c}}$ \\ Anna Gennadjevna Orlova, ${ }^{d}$ Pavel Vladimirovich Subochev, ${ }^{d}$ Dmitriy Vladimirovich Safonov, ${ }^{\mathrm{b}}$ \\ Natalia Mikhailovna Shakhova, ${ }^{d}$ and Anna Vladimirovna Maslennikova ${ }^{b, e}$ \\ a Volga Region Medical Center, Nizhny Novgorod, Russia \\ bNizhny Novgorod State Medical Academy, Nizhny Novgorod, Russia \\ ${ }^{\mathrm{c} R e g i o n a l}$ Oncological Hospital, Nizhny Novgorod, Russia \\ 'Institute of Applied Physics RAS, Nizhny Novgorod, Russia \\ eLobachevsky University, Nizhny Novgorod, Russia
}

\begin{abstract}
The ability for noninvasive visualization of functional changes of a tumor's oxygenation and circulatory system offers new advantages for prognosis and monitoring of the treatment efficacy. The results of breast cancer oxygen state study under chemotherapy action obtained by diffuse optical spectroscopy (DOS) in combination with Doppler ultrasonic imaging are presented. Complex use of optical and ultrasound methods gives complementary information about the size of the tumor node, peculiarities of its vascular bed, rate of its blood flow as well as oxygenation, and provide a picture of the tumor response to treatment. Comparison with tumor pathologic response allowed to identify differences in the changes of these parameters depending on the degree of pathological tumor response to chemotherapy. It was demonstrated that fourth and fifth degrees of therapeutic pathomorphism may be predicted by the increase of oxygen saturation level after the first cycle of chemotherapy. If the reduction or absence of the oxygen saturation dynamics is observed, first or second degree of pathological tumor response can be expected. Additional ultrasound investigation of the tumors may be useful for observation of the dynamics of tumor blood flow thereby for understanding the reasons of induced chemotherapy oxygenation changes. The proposed approach based on DOS and ultrasonography may be applied for monitoring of breast tumors under therapy and prediction of their sensitivity. ๑ 2018 Society of Photo-Optical Instrumentation Engineers (SPIE) [DOI: 10.1117/1.JBO.23.9.091410]
\end{abstract}

Keywords: breast cancer; neoadjuvant chemotherapy; pathological response; diffuse optical spectroscopy; tumor oxygen state. Paper 170816SSR received Dec. 20, 2017; accepted for publication Apr. 23, 2018; published online May 15, 2018.

\section{Introduction}

Breast cancer $(\mathrm{BC})$ is a leading oncologic disease of active working age, being also the main malignant tumor mortality cause among women. In accordance with the data of the International Agency for Research on Cancer, BC is the most common cancer in women worldwide representing about $12 \%$ of all new cancer cases and $25 \%$ of all cancers in women. ${ }^{1}$ Since a systemic model of BC pathogenesis had been accepted, ${ }^{2,3}$ a substantial enhancement of the role of chemotherapy (CT) and hormonal therapy for its treatment has occurred. ${ }^{4}$ Neoadjuvant CT has been initially considered as a method of "downstaging" of locally advanced tumors prior to surgery; 5,6 however, in the past years, the indications for it were widely extended. ${ }^{7}$ The response of breast tumors to the neoadjuvant $\mathrm{CT}$ is considered to be a surrogate biomarker, allowing to predict prognosis and outcome of the disease. ${ }^{8}$ The best marker of therapeutic efficiency having influence of the general and recurrencefree survival is the pathological complete tumor response (pCR), which is defined as absence of viable tumor cells in the breast tissue and regional lymph nodes. ${ }^{7,9}$ However, pCR is only achieved in $\sim 13 \%$ to $33 \%$ of the patients, due to the grade of

*Address all correspondence to: Mikhail Viktorovich Pavlov, E-mail: pavlov. med88@gmail.com malignancy, tumor immunophenotype, and the CT scheme. ${ }^{5,10,11}$ Moreover, in a certain percentage of the patients, the preoperative CT shows an absence (grade I or II) of pathological tumor response. ${ }^{12-16}$ In this case, problems occur connected with a delay of surgery, development of toxicity, and high cost of the actually ineffective therapy. ${ }^{15}$ From this point of view, development of methods allowing for early prognosis of the tumor response after the beginning of $\mathrm{CT}$ is of great importance.

Methods of structural imaging [ultrasound investigation (USI), x-ray mammography, magnetic resonance imaging (MRI)] that allow detecting dimensional changes of the lesion ${ }^{16}$ are not suitable for early assessment of the tumor response to the administered therapy. In the past years, active studies have been conducted to use metabolic imaging methods (MRI and positron emission tomography $)^{17-19}$ for such a purpose, as well as studies have been started for the investigation of opportunities provided by optical methods for assessment and monitoring of the oxygen status of biological tissues. ${ }^{20,21}$ The latter are based on the calculation of optical parameters of biological tissues (scattering and absorption) using multiple-scattering light interacting with the object. ${ }^{22-26}$ The experiments conducted by the method of diffuse optical spectroscopy (DOS) have shown that CT, dependent on its efficiency, leads to a certain change of parameters

1083-3668/2018/\$25.00 ๑ 2018 SPIE 
of neoplasm's oxygen saturation. ${ }^{27}$ The results build a basis for use of optical methods in clinical research. ${ }^{28,29}$ The papers ${ }^{30-32}$ summarize the results of oxygen status monitoring of breast tumors in the course of neoadjuvant CT by means of DOS. The studies conducted enabled offering of criteria of early tumor response. As such predictors, the initial (basic) level of total hemoglobin, ${ }^{33}$ the initial level of oxyhemoglobin, ${ }^{34}$ the trend of changes of the total hemoglobin after the first CT cycle,$^{35}$ dynamics of tumor saturation, ${ }^{36}$ and the initial level of deoxyhaemoglobin ${ }^{37}$ were suggested.

Along with the oxygen saturation of the tumor and its dynamics in the course of the therapy, an important parameter is the tumor vascularity that determines the oxygen delivery to the tissue. Its monitoring during a treatment might provide valuable additional information concerning tumor response to therapy. ${ }^{38}$ However, tumor hemodynamics evaluation over the course of cancer therapy is limited in a routine clinical practice because of the lack of appropriate and convenient technologies. Several methods exist for the measurement of tumor hemodynamics, such as optoacoustic imaging ${ }^{39}$ and optical coherence tomography angiography. ${ }^{40}$ The latter's limitation is the imaging depth (the instrumentation may be used only in case of superficial neoplasms). Nowadays, a prospective technology for microvasculature blood flow measurement, namely, diffuse correlation spectroscopy (DCS), that uses the temporal fluctuations of near-infrared light to measure blood flow has been developed. ${ }^{41-43}$ The main advantage of the method is its opportunity to study deep-seated tissues such as brain ${ }^{43}$ and muscle. ${ }^{44}$ The method was also used for therapeutic monitoring of the (chemo)radiation therapy of head/neck cancer and for prediction of tumor response to treatment, ${ }^{41,45}$ demonstrating a predictive ability in revealing the tumors sensitive and nonsensitive to the treatment.

A standard method of blood flow detection, color Doppler USI, allows evaluating relatively large vessels. A combination of USI and DOS has been used for studies of nonhomogeneous oxygen saturation and vascularization of large breast tumors. ${ }^{46}$ The paper ${ }^{47}$ presents the dynamics of tumor blood flow in patients to whom neoadjuvant CT for BC was administered. Divergent changes of this parameter were detected, dependent on the degree of tumor response, as well as a considerable "delay" of the dimensional and the vasculature changes in the tumor. Color Doppler ultrasonography was used to monitor the response to therapy and to identify possible correlations between survival and various Doppler indices (resistivity index, pulsatility index, and maximum flow velocity) in Refs. 48 and 49. The pre- and post-CT indices were compared with each other in order to assess and evaluate the response to $\mathrm{CT}$.

The study objective was investigation of a predicting ability of early changes of breast tumor oxygenation (by DOS) and tumor vascularity (by Doppler ultrasonic imaging) in the course of neoadjuvant polychemotherapy.

\section{Materials and Methods}

\subsection{Patient Data}

The study was performed in Nizhny Novgorod Regional Clinical Oncologic Hospital. Between November 2015 and May 2017, 25 patients with locally advanced BC were enrolled. Patients had no history of prior malignant neoplasms and had not received prior CT. Performance status was $0-1$ according to the Eastern Cooperative Oncology Group scale. Patient informed consent and study methods were reviewed by the Research Ethics Border of the Nizhny Novgorod State Medical Academy. The criteria of participation were histologically confirmed BC stage II to IV; signed informed consent of the patient for participation in a clinical study; scheduling of CT in a combined therapy of BC. Patients with tumors larger than $8 \mathrm{~cm}$, skin-piercing tumors, and age under 18 were excluded. Thirty patients were enrolled in the study according to the

Table 1 General description of patients included in the patients group.

\begin{tabular}{|c|c|c|}
\hline Description & & $\begin{array}{l}\text { Number of } \\
\text { patients } \\
\text { (percentage) }\end{array}$ \\
\hline \multirow[t]{4}{*}{ Age } & $<40$ & $4(16 \%)$ \\
\hline & 41 to 50 & $8(32 \%)$ \\
\hline & 51 to 60 & $8(32 \%)$ \\
\hline & $>61$ & $5(20 \%)$ \\
\hline \multirow[t]{6}{*}{ BC stage } & Ila & $2(8 \%)$ \\
\hline & $\mathrm{llb}$ & $3(12 \%)$ \\
\hline & Illa & $9(36 \%)$ \\
\hline & Illb & $7(28 \%)$ \\
\hline & IIlc & $3(12 \%)$ \\
\hline & IV & $1(4 \%)$ \\
\hline \multirow{2}{*}{$\begin{array}{l}\text { State of regional } \\
\text { lymph nodes }\end{array}$} & No metastases & $4(16 \%)$ \\
\hline & With metastases & $21(84 \%)$ \\
\hline \multirow[t]{5}{*}{ Immunophenotype } & Luminal A & $5(20 \%)$ \\
\hline & Luminal B HER2neu-negative & $11(44 \%)$ \\
\hline & Luminal B, HER2neu-positive & $2(8 \%)$ \\
\hline & HER2neu-positive & $3(12 \%)$ \\
\hline & Triple negative & $4(16 \%)$ \\
\hline \multirow[t]{3}{*}{ Scheme of $\mathrm{CT}$} & $\begin{array}{l}\text { Doxorubicin and } \\
\text { cyclophosphamide (AC) }\end{array}$ & $16(64 \%)$ \\
\hline & $A C+$ paclitaxel & $4(16 \%)$ \\
\hline & $A C+$ trastuzumab & $5(20 \%)$ \\
\hline \multirow{5}{*}{$\begin{array}{l}\text { Degree of } \\
\text { pathological } \\
\text { tumor response }\end{array}$} & I & $4(16 \%)$ \\
\hline & II & $5(20 \%)$ \\
\hline & III & $7(28 \%)$ \\
\hline & IV & $6(24 \%)$ \\
\hline & V & $3(12 \%)$ \\
\hline Total & & 25 (100\%) \\
\hline
\end{tabular}


inclusion criteria. Twenty-five of them completed the treatment and were included in the analysis. Five patients did not complete the full course and were excluded. The scheme and number of cycles of CT were prescribed depending on tumor immunophenotype, tumor's stage, and patient's age. Sixteen patients received a CT regimen that consisted of doxorubicin and cyclophosphamide (AC regimen). This treatment was followed sequentially with paclitaxel in patients with triple-negative BC (four patients). Five patients with luminal B HER2neu-positive and HER2neu-positive $\mathrm{BC}$ were treated with doxorubicin, cyclophosphamide, and trastuzumab. The summary is provided in Table 1.

\subsection{Study Design}

Mammography, ultrasonic breast and peripheral lymph node imaging, tumor biopsy with determining of receptor status, HER2neu status, and proliferation index of all patients were made before the therapy. The assessment of the oxygen saturation level of the tumor by DOS and of the blood flow of the tumor area by Doppler scanning were conducted prior to CT start and prior to the second CT cycle. Study design is presented in Fig. 1.

After CT, surgery was carried out with subsequent pathological study of the tumor and assessment of the pathological tumor response to the therapy. Based on the pathological results, the patients were divided into groups of complete and partial response, as well as those with absence of tumor response to the therapy. The degree of pathological tumor response was determined in accordance with Ogston et al. ${ }^{50}$

\subsection{Diffuse Optical Spectroscopy}

Experiments on DOS were performed on the experimental setup with parallel plane geometry developed at the Institute of

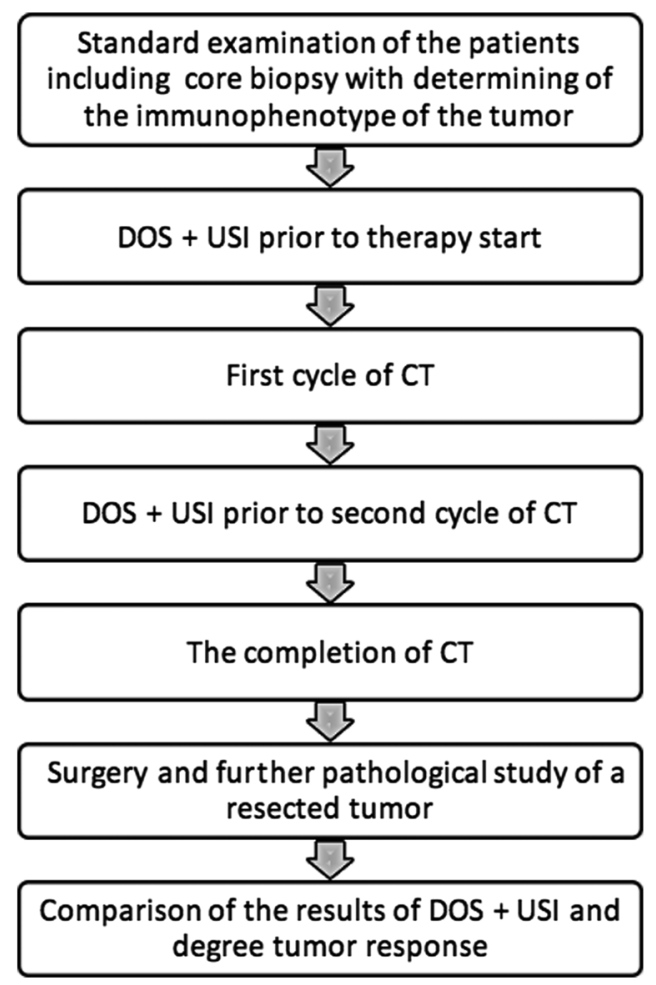

Fig. 1 Study design.
Applied Physics, RAS (Nizhny Novgorod, Russia). ${ }^{26}$ Three laser fibers coupled in a bundle illuminated the studied volume at three wavelengths: $684 \mathrm{~nm}$ corresponding to the maximum absorption of deoxygenated hemoglobin, $850 \mathrm{~nm}$ corresponding to maximum absorption of oxyhemoglobin, and $794 \mathrm{~nm}$ isosbestic point. In this device, a high-frequency amplitude modulation $(140 \mathrm{MHz})$ was used, allowing determination of absorption coefficients more accurately due to separate determination of scattering and absorption coefficients. The diffuse light was detected by the Hamamatsu photomultiplier tube with an automatic gain control. The images were obtained by simultaneous scanning of the source and the detector located along the sagittal axis from the opposite sides of the studied subject with a step of 1 to $2 \mathrm{~mm}$ synchronously. In each position, data were obtained from all three sources (Fig. 2).

For scanning, a patient was lying in a prone position with a breast between two sliding transparent plates preventing its contact with the source and the detector, slightly pressed for equal thickness of the breast in the scanning area. The distance between the plates was fixed. The scanning area selection was based on the results of the clinical examination and the mammographic data. The local signal change allowed for detection of optical nonuniformity in the scanned area for subsequent visual check of the scanning area.

\subsection{Numerical Analysis of DOS Images}

The DOS images obtained during the scanning contained the amplitude and the phase of the photon density waves of the signal which passed through the tested tissue for three wavelengths. Based on the initial data, two-dimensional images of distribution of reconstructed absorption and reduced scattering at three wavelengths using MathCAD software were obtained. The obtained values of coefficients were used for calculation of oxy- and deoxyhemoglobin concentrations. ${ }^{26}$ Based on concentration data of these compounds, the equation $\mathrm{tHb}=\left[\mathrm{HbO}_{2}+\right.$ $\mathrm{HHb}$ ] was used for total hemoglobin contents, whereas the equation $\mathrm{StO}_{2}=\left[\mathrm{HbO}_{2}\right] /\left[\mathrm{HbO}_{2}+\mathrm{HHb}\right] \times 100 \%$ was used to determine the level of blood oxygen saturation in the region of interest. ${ }^{32}$ Earlier study showed that blood saturation values calculated based on the DOS correctly depict the oxygenation value of the tissue. ${ }^{20}$ For further analysis and determining the parameters of the oxygen status of the tumor tissue ImageJ software (NIH) was used. The region of interest was contoured manually, overlapping the tumors in mammograms and DOS images, also considering US images [Fig. 2(b)]. The contouring of images obtained after the first CT cycle was conducted under recognition of the dimensional changes of the tumor node in accordance with the data of the standard ultrasonic investigation. ${ }^{51}$

\subsection{Doppler Ultrasonic Imaging}

For assessment of the blood flow status of the breast, an original method of ultrasonic investigation was elaborated. The scanning of the interest area was performed by "Medison Accuvix-V20" (Samsung, Korea) with a multifrequency linear sensor 5.0 to 13.0 $\mathrm{MHz}$ in the energetic Doppler mode with 5-mm pitch in mutually vertical planes. The center of the tumor was overlapped with the center of the mask of transparent polymer material with dot-shaped holes in mesh pattern every $5 \mathrm{~mm}$ for marker points of the skin [Fig. 2(c)]. On these dots, after mask removal, serial scanning of the whole tumor in mutually vertical planes in energetic Doppler mode was performed, with a 
(a)

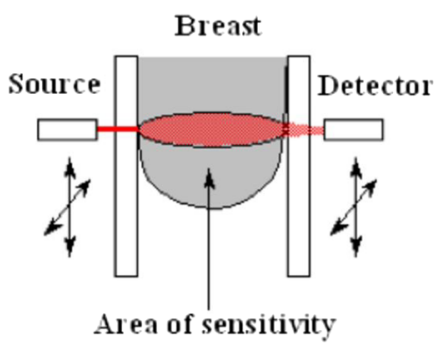

(b)

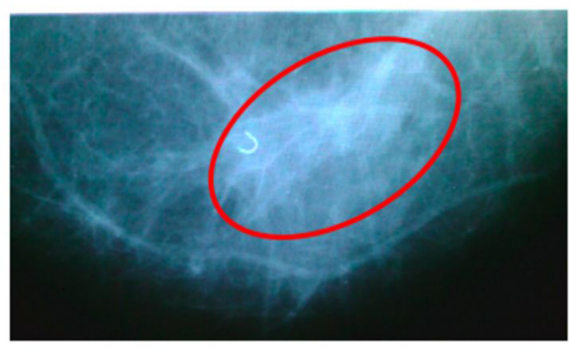

$\mathrm{StO}_{2}(\%)$

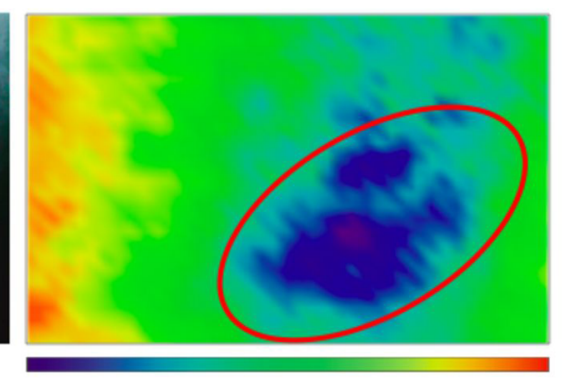

70

80

(c)
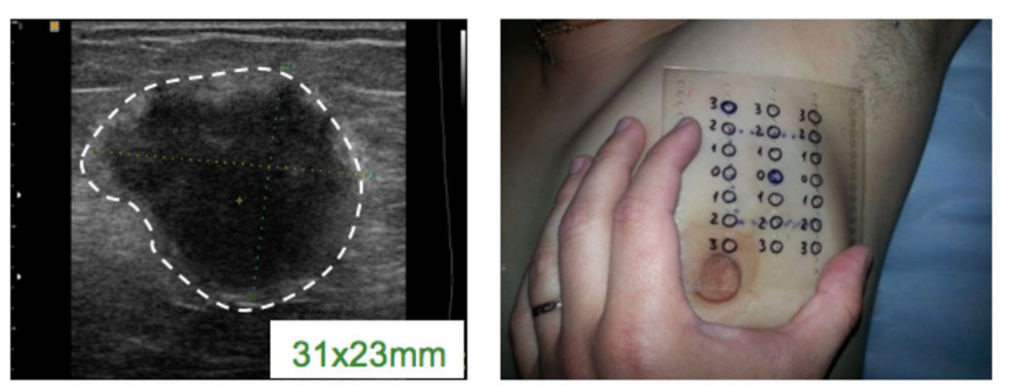

Fig. 2 Investigation methods: (a) arrangement of the source and the detector; (b) example of an x-ray mammogram and DOS image of BC (the red oval line marks the tumor area). The dimension of DOS image is $6 \times 8 \mathrm{~cm}$. (c) A sample of US image and breast skin marking in the tumor projection for ultrasonic scanning by means of a mask.

set dimension of the interest window. Thereby, if the tumor size is less than $4 \mathrm{~cm}$, the sufficient number of scans shall be 18 ( 9 in sagittal and 9 in axial planes). In case the tumor is bigger than $4 \mathrm{~cm}$, the mask may help to add new points required for scanning of the whole area of the tumor tissue. By means of MATLAB $^{\circledR}$ (MathWorks, Natick, Massachusetts), in each plane a percentage of the colored pixels number (equivalent to blood vessels) to the total pixel number of the image ${ }^{52}$ [Fig. 5(c)] was determined. All images were analyzed during the tumor scanning, the obtained data were summarized, and an average value was calculated to quantify the vasculature of the tumor. The vascularization of the tumor was evaluated by this method prior to therapy start and to the second cycle of CT. Then, a difference of the value before and after the first CT cycle was determined in percent. This difference (under recognition of the direction of the change) was accepted as the basic parameter characterizing the effect produced on the vasculature of the tumor by the therapy.

\subsection{Statistical Analysis}

A correlation analysis between changes of tumor oxygenation and tumor blood flow after $\mathrm{CT}$ beginning, $\mathrm{BC}$ stage, tumor immunophenotype, and tumor pathologic response was performed by GraphPad Prism (Graphpad Software Inc., La Jolla, California). A correlation cloud was created and linearly approximated by Spearman's rank correlation coefficient to confirm the hypothesis of correspondence between these indicators and tumor response. $P$ value $<0.05$ was considered to be statistically significant. For more accurate calculation, a number of dead cells in the residual tumor node detected after surgery by a pathologist were used as a numerical criterion of tumor response besides the attribution of tumor response to one of five subgroups according to Ogston et al. ${ }^{50}$

\section{Results}

Breast tumors have demonstrated diverging dynamics of the oxygen saturation level dependent on pathological tumor response to CT [Fig. 3(a)]. In case of degree IV and V of pathological tumor response (massive extinction of invasive cells, $>90 \%$ of cell loss), an enhancement of the oxygen saturation of the tumor referring to the initial value after the first cycle of CT was observed in eight patients of nine. The lowering of the level of oxygen saturation by $8 \%$ was detected in one patient with IV degree of pathological tumor response [Fig. 3(a) (yellow and green columns)].

In case of the first and the second degrees of pathological tumor response (hard-to-notice separate tumor cells, but without their number going back), a lowering (in six patients) or absence of dynamics (in two patients) of the oxygen saturation level of the tumor in comparison with the initial one was observed in eight of nine patients [Fig. 3(a) (blue and orange columns)]. In one patient with the II degree of pathological tumor response to therapy, enhanced level of oxygen saturation by $12 \%$ as compared with the initial level was observed.

In case of the third pathological tumor response degree (reduction in tumor cells of between $30 \%$ and $90 \%$ ), both enhancement and decrease of the oxygen saturation level of the neoplasm was observed. In three patients, enhancements of the saturation were detected, also one significant enhancement value of $57 \%$. In two cases, a decrease was observed and in the other two no oxygen saturation level changes were detected after the first CT cycle [Fig. 3(a) (gray columns)].

The analysis of the dynamics of the blood flow through the tumor could not show a significant dependence between the changes of this parameter after the first CT cycle and the degree of pathological tumor response. In most cases, a decrease of the blood flow rate was observed, sometimes significant and sometimes negligible [Fig. 3(b)]. In four patients with different 
(a)

Pathological tumor response (25 patients)

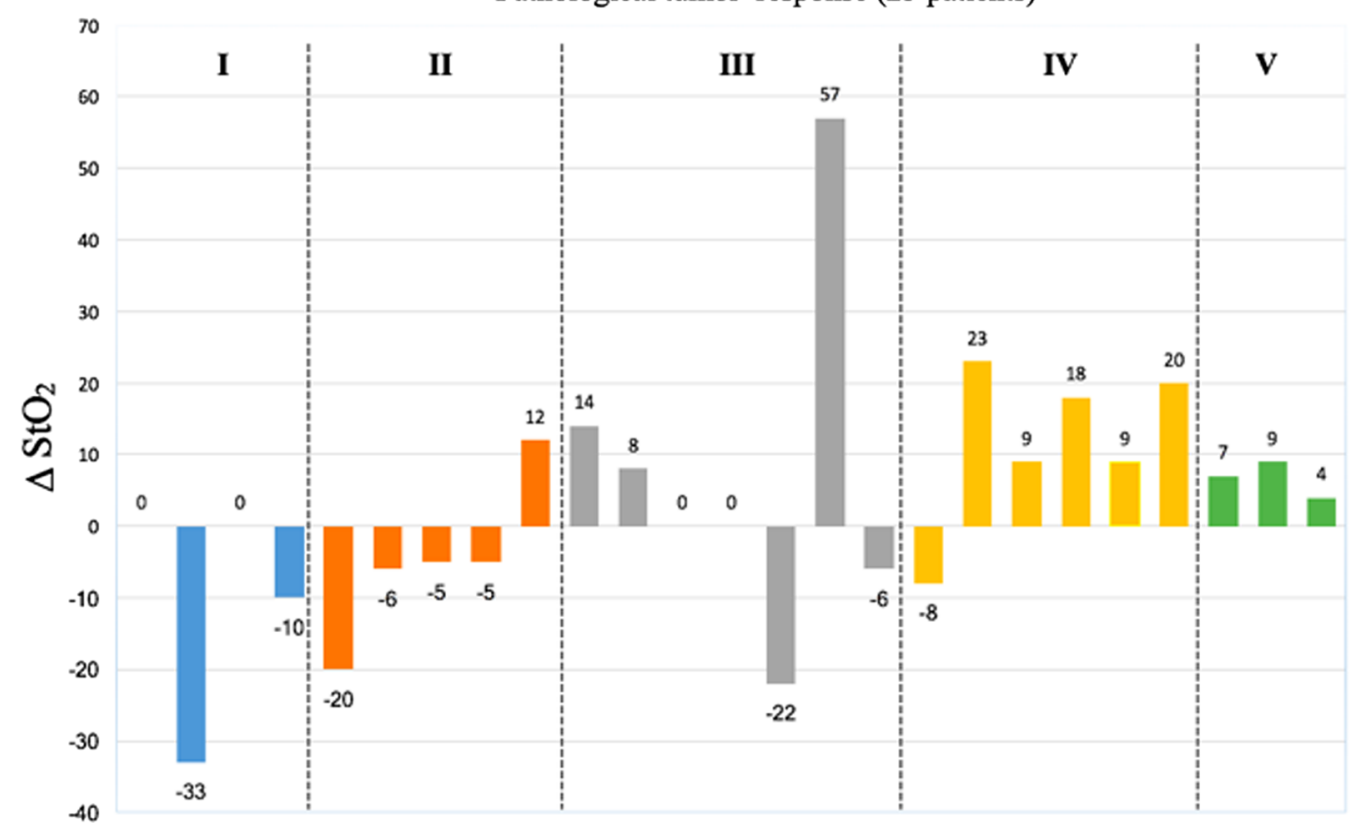

(b)

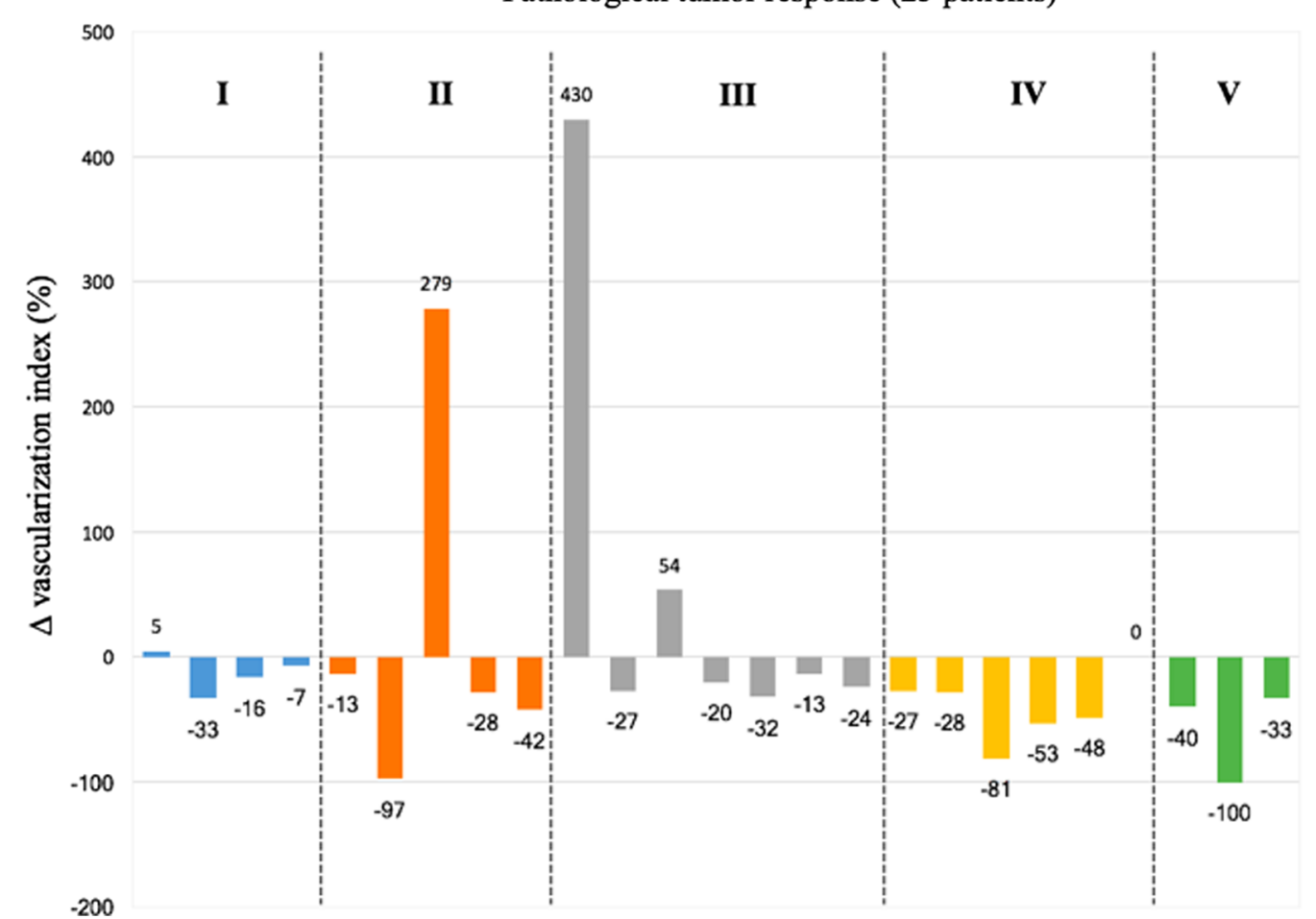

Fig. 3 Oxygen saturation and blood flow rates of the tumor after the first cycle of CT, dependent upon the degree of pathological tumor response (25 patients). (a) DOS data and (b) USI data.

degree of pathological tumor response, increased blood flow ratings of the neoplasm were observed, in one case, more than a fourfold increase [Fig. 5(c)]. Obviously, the blood flow rating within the tumor area might be influenced by processes not directly connected with the death of the neoplasm's cells or with the inhibition of the tumor neoangiogenesis under the influence of CT. It might be associated with the development of the inflammation in the peritumoral area. To illustrate the obtained results, see the following clinical examples.
A comparison of the indicators of tumor oxygenation and tumor response revealed a moderate but significant positive correlation between the analyzed parameters [Fig. 4(a)]. Linear fitting of the experimental data provided a satisfactory value of approximation reliability $\left(R^{2}>0.34\right)$ and moderate Spearman's rank correlation coefficient $(r=0.628)$ with a significance level $p=0.0003$. We failed finding a statistically significant correlation between the number (percentage) of dead cells and blood flow changes $(r=-0.394, p=0.051), \mathrm{BC}$ 

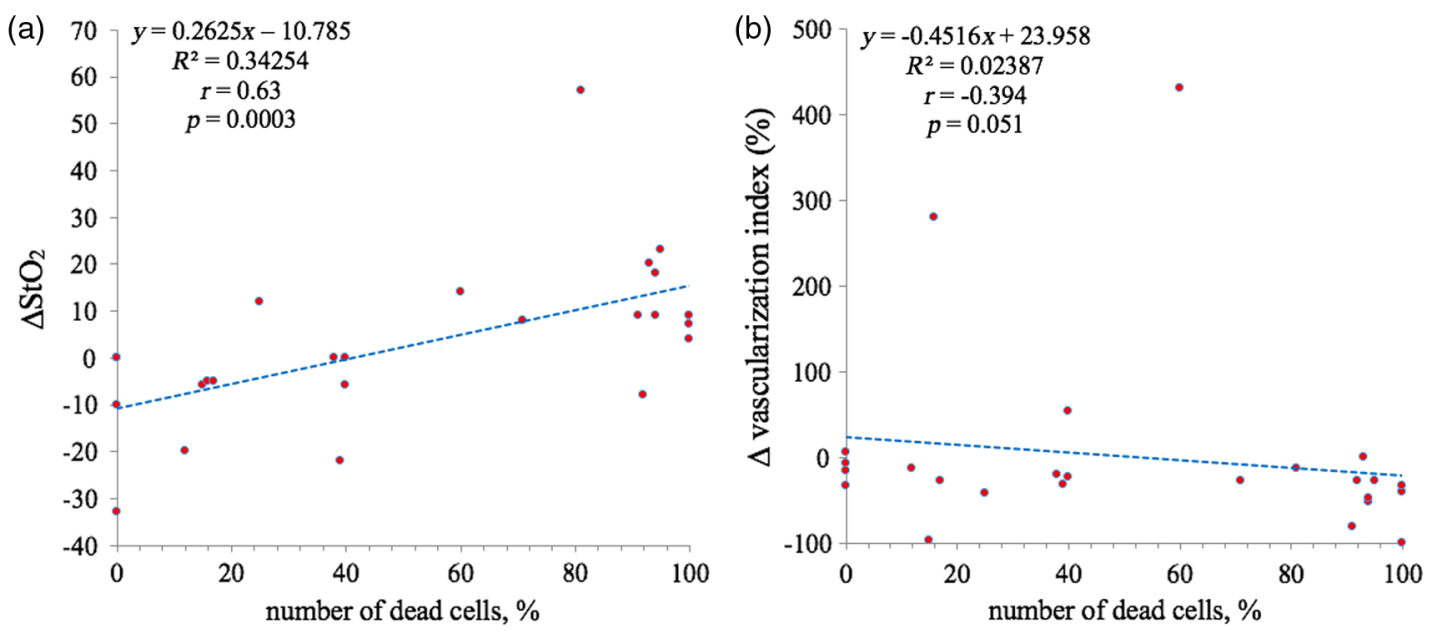

Fig. 4 A correlation between pathological tumor response (the number of dead cells after CT completion) and (a) $\Delta \mathrm{StO}_{2}$ and (b) $\Delta$ vascularization index (\%) after the first cycle of CT.

stage $(r=-0.199, \quad p=0.340), \quad$ and immunophenotype $(r=0.267, p=0.198)$.

Case report 1. Patient A, age of 53, was administered neoadjuvant $\mathrm{CT}$ (schedule $\mathrm{AC}$ ) due to triple-negative left $\mathrm{BC}$ of stage 2a (T2N0M0). During the ultrasonic investigation prior to neoadjuvant $\mathrm{CT}$, a tumor node with uneven and vague contour $41 \times 41 \mathrm{~mm}$ was detected, with necrosis in the center and poor peripheral blood flow [Figs. 5(b) and 5(c), top image]. The pathologic investigation showed vast layers of necrotic tissue with a small layer of invasive BC with crash-syndrome phenomenon [Fig. 5(d) (top image)].

In DOS images after the first CT cycle, an enhancement of level of oxygen saturation was detected by $14 \%$ [Fig. 5(a)] as compared with the initial value, allowing to suppose a good response of the tumor to the administered therapy. In accordance with the ultrasonic investigation, an increase of the tumor size (up to $49 \times 48 \mathrm{~mm}$ ) was detected, along with a considerable growth of the central necrosis area and occurrence of a distinct edema of perinodular tissue, which is equal to the development of an inflammation process around the tumor node [Fig. 5(b), bottom image]. In addition, a significant (by $430 \%$ as compared with the initial level) vascularization growth in the tested area was detected. The blood vessels appeared all-over the peripheral area of the tumor tissue (around the necrotic area) and in the perinodular area [Figs. 5(c) and 5(d)]. After four CT cycles, the patient underwent mastectomy. During the pathological investigation, the III degree of pathological tumor response after Ogston et al. was detected. The central part of the tumor node was occupied by a vast necrosis area [Fig. 5(d) (bottom image)]. Having analyzed the results of the pathologic investigation after core-biopsy and of the removed tumor, we suppose association of the dimensional growth of the tumor blood vessels with the vast necrosis area in the center of the tumor node. Thus, in this case, the vascularization has grown (a)

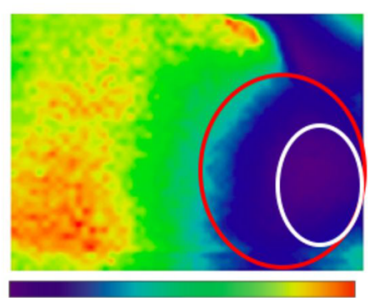

0

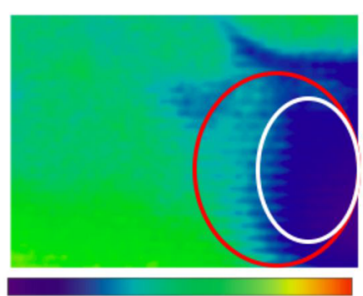

(b)
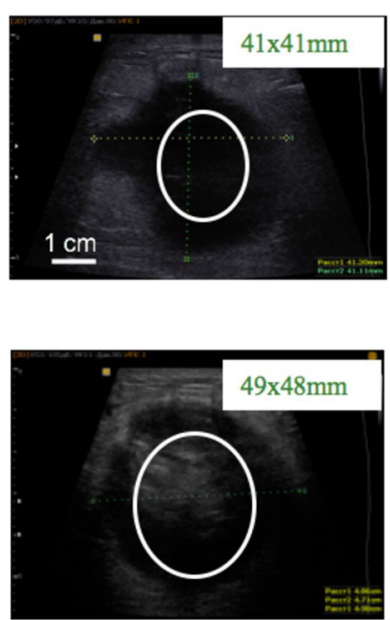

(c)

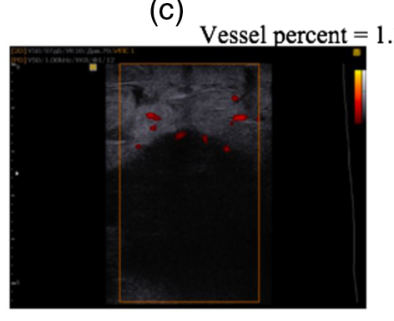

Vessel percent $=6.3$

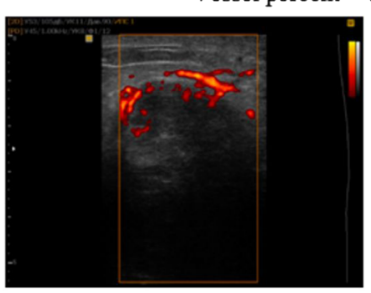

(d)
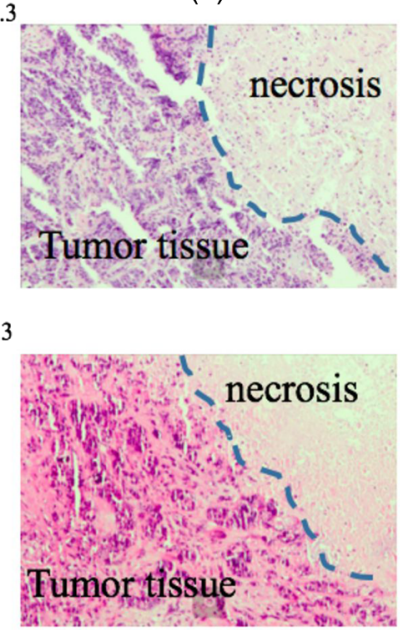

0

100

Fig. 5 Dynamics of tumor oxygen saturation and blood flow (patient A) (a) DOS imaging $\left(\mathrm{StO}_{2}\right)$ with tumor area marked by red oval, area of necrosis marked by white oval. (b) USI imaging (B-mode), area of necrosis marked by white oval. (c) US imaging (power Doppler mode). (d) Histologic samples (after core-biopsy, top image; postoperative material, bottom image). Top row, results prior to CT; bottom row, after $(\mathrm{a}-\mathrm{c})$ the first CT cycle or (d) on accomplishment of CT. DOS image dimensions $6 \times 8 \mathrm{~cm}$. 
due to the presence of an inflammation process in the peritumoral area stipulated by the vast necrosis area, however, not for the reason of the absence of response to the administered therapy.

Case report 2. Here is the example of changes in the tumor oxygen saturation and blood flow of patient $\mathrm{E}$, age of 45 . Diagnosis: right $\mathrm{BC}$, stage IIa (T2NOM0), HER2neu-positive cancer (estrogen receptors 0 points, progesterone receptors 0 points, Ki67 40\%). During ultrasonic investigation, prior to administering of neoadjuvant therapy, a tumor node was detected in the breast with uneven and vague contour with dimensions $28 \times 14 \mathrm{~mm}$ [Fig. 6(b)]. The pathomorphology showed invasive ductal carcinoma [Fig. 6(d) (top image)]. The initial level of vascularization was $5.3 \%$. The patient was assigned neoadjuvant polychemotherapy (AC + trustuzumab schedule). After the first cycle of CT, a tumor shrinked to $18 \times 10 \mathrm{~mm}$ [Fig. 5(b)], oxygen saturation enhancement by up to $7 \%$ after the results of DOS [Fig. 6(a)] and reduction of the tumor blood flow by $40 \%$, as compared with the initial values [Fig. 6(c)]. A pathologic investigation after six CT cycles and surgery failed to find vital tumor cells, equivalent to the $\mathrm{V}$ degree of pathological tumor response [Fig. 6(d) (bottom image)].

Case report 3. Here is the example of changes in the oxygen saturation and blood flow of breast tumor of patient C, age of 50 . (a)
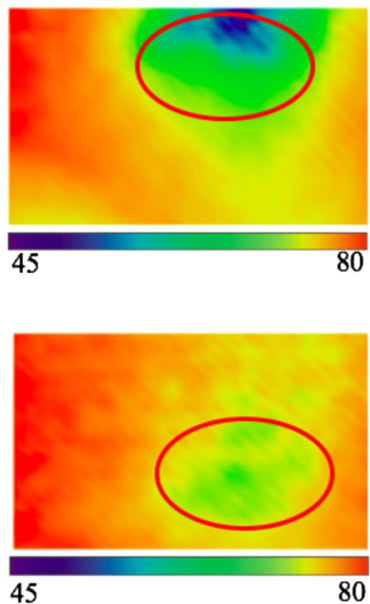

(b)
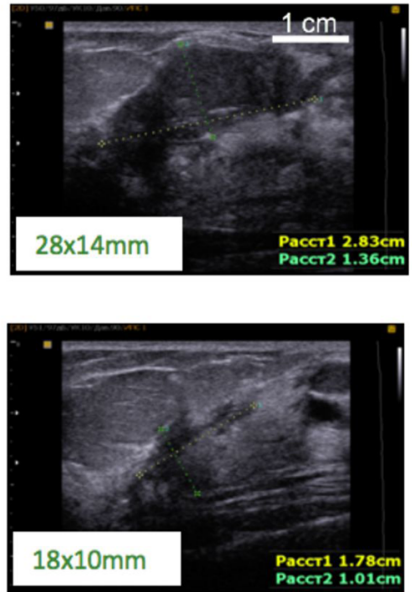

(c) Vessel percent $=5.29$

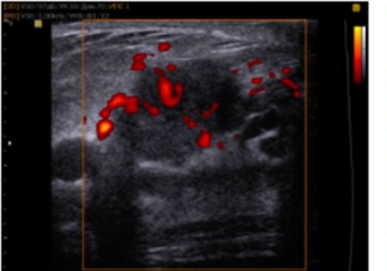

Vessel percent $=0.16$

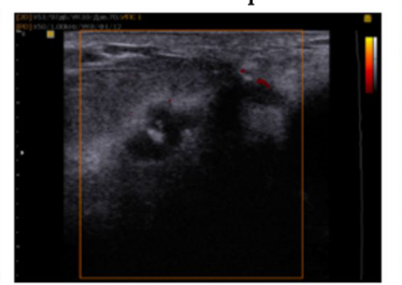

(d)
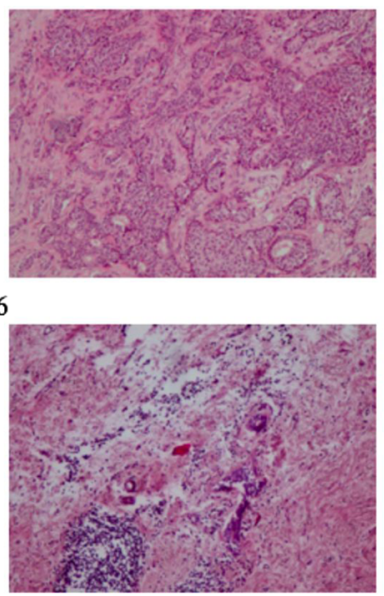

Fig. 6 Dynamics of tumor oxygen saturation and blood flow (patient E) (a) DOS imaging $\left(\mathrm{StO}_{2}\right)$ with tumor area marked by red oval. (b) USI imaging (B-mode). (c) US imaging (power Doppler mode). (d) histologic samples (after core-biopsy, top image; postoperative material, bottom image). Top row, results prior to $\mathrm{CT}$; bottom row, after $(\mathrm{a}-\mathrm{C})$ the first CT cycle or (d) on accomplishment of CT. DOS image dimensions $6 \times 8 \mathrm{~cm}$.

(a)
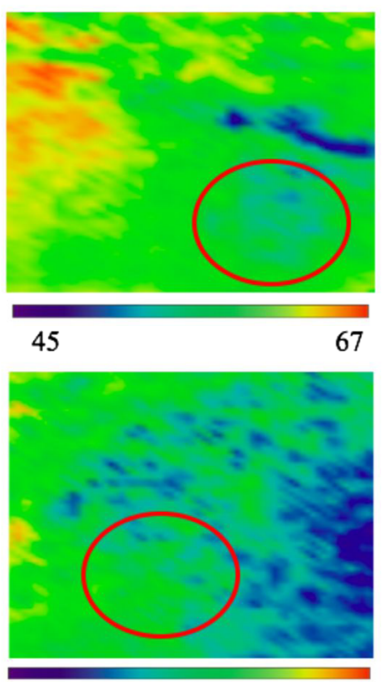

45

67 (b)
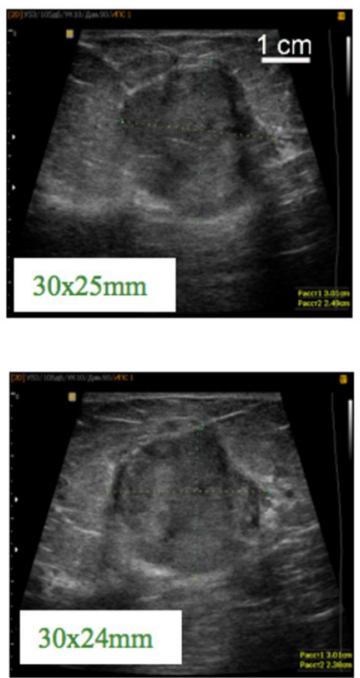

(c) Vessel percent $=7.01$
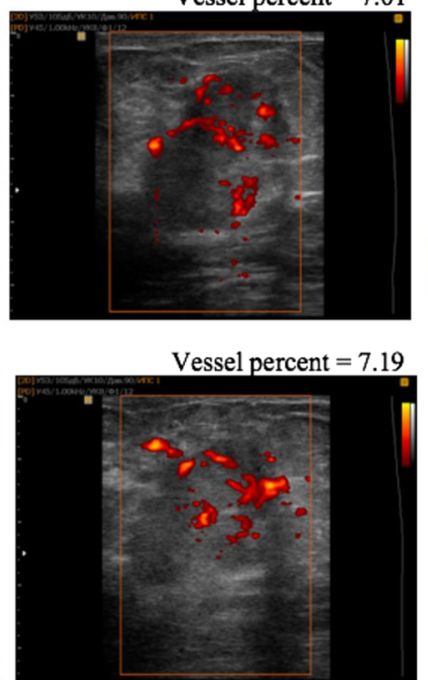

(d)
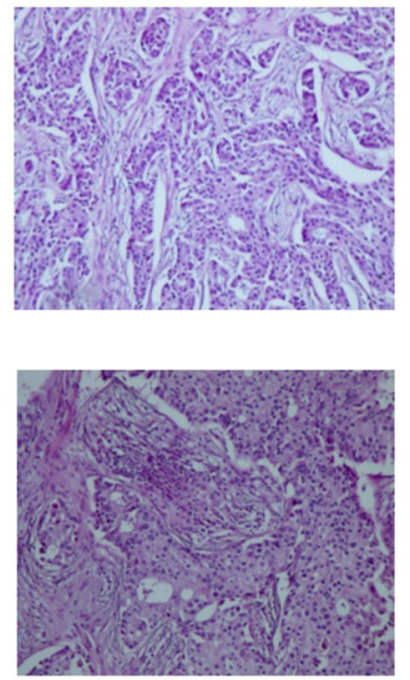

Fig. 7 Dynamics tumor oxygen saturation and blood flow (patient C) (a) DOS imaging $\left(\mathrm{StO}_{2}\right)$ with tumor area marked by red oval. (b) USI imaging (B-mode). (c) US imaging (power Doppler mode). (d) histologic samples (after core-biopsy, top image; postoperative material, bottom image); top row, results prior to CT; bottom row, after the $(a, b, c)$ first CT cycle or (d) on accomplishment of CT. DOS image dimensions $6 \times 8 \mathrm{~cm}$. 
Diagnosis: left BC, stage IIIa (T2N2M0), luminal B HER2neunegative cancer (estrogenic receptors eight points, progesterone receptors six points, Ki67 30\%). Prior to neoadjuvant therapy, a node with an uneven and vague contour with dimensions of $30 \times 25 \mathrm{~mm}$ was detected in the breast [Fig. 7(b)]. The pathologic test showed invasive ductal carcinoma [Fig. 7(d) (top image)]. The initial level of vascularization was $6.9 \%$. The patient was assigned neoadjuvant polychemotherapy (AC schedule). After the first cycle of CT, the size of the tumor node remained unchanged [Fig. 7(b)]. Based on the results of DOS, the oxygenation level was unchanged [Fig. 7(a)], the tumor blood flow increased by 5\% [Fig. 7(c)]. A pathologic investigation after six CT cycles and a mastectomy detected the I degree of pathological tumor response, equal to therapy failure [Fig. 7(d) (bottom image)].

\section{Discussion}

Since the mid-2000s, there were attempts to use various modifications of optical diffuse spectroscopy as a method to predict the sensitivity of BC to CT. At the base of all suggested predictive criteria were indicators of the oxygen status of the breast tumor and/or their changes in the course of treatment. ${ }^{32,51}$ When planning the study, we expected that the oxygenation level of any tissue reflects the ratio of oxygen delivery and oxygen consumption by tissues, i.e., tumor tissue. ${ }^{53,54}$ The disproportion between the escalating demands of the tumor tissue in oxygen and its inadequate delivery of the pathological vascular bed leads to the phenomenon of tumor hypoxia that is known to be a characteristic feature of many solid tumors. ${ }^{55}$ In our study, the difference between the tumor oxygenation before and after the first course of treatment was chosen as the main criterion, which reflects the tumor sensitivity to CT. When analyzing the dependence of changes in this parameter on the pathologic tumor response, a moderate but statistically significant correlation was revealed $(r=0.628, p=0.0003)$. The trend of changes in tumor oxygenation was different depending on the effect of the treatment. Most tumors that appeared to be sensitive to CT (IV and V degrees of pathologic response that corresponds to $90 \%$ up to $100 \%$ of dead tumor cells) have demonstrated improved oxygenation in 3 weeks after treatment beginning compared to baseline. The changes of the oxygen saturation level of tumor tissue may depict changes of oxygen consumption by the tumor as a result of mortality of a certain part of the tumor cells. ${ }^{56}$ In this case, it is possible to draw an analogy with the process of tumor reoxygenation after fractionated radiation therapy (4R of radiotherapy). ${ }^{57}$ Tumor tissue reoxygenation appears because of decrease of the number of viable tumor cells after irradiation, and, accordingly, the decrease of tumor oxygen demands. ${ }^{58}$ In case of ineffective CT, in most cases there was a decrease in the level of tumor oxygenation or lack of its dynamics. From our point of view, this indicates the preservation or increase of oxygen consumption by a tumor tissue, i.e., the preservation of the number of viable tumor cells.

In most works devoted to the predictive criteria for the response of breast tumors to $\mathrm{CT}$, the tumors are divided to the subgroups of complete (pCR) or noncomplete (non-pCR) response. Subgroup of non-pCR aggregates tumors with grade 1 (the absence of cell's loss), grade 2 (minimal cell's loss), grade 3 (cell's loss 30\% to 90\%), and grade 4 (more than $90 \%$ of dead cells), i.e., is very heterogeneous in respect of biological characteristics. ${ }^{51}$ The authors underscore that a more refined clinical assessment of the non-pCR group using a continuously variable endpoint, such as the residual cancer burden or the Miller-Payer system, could lead to a better understanding of the non-pCR changes, and to a better separation between response groups.

The same approach was used in Ref. 59, when evaluating a predictive value of an initial level of total hemoglobin of tumor tissue before treatment and its changes after three cycles of CT. Tumors with grades 1 to 3 and 4 to 5 were compared. We assume that the evaluation of tumor oxygenation in case of grade 3 is the most difficult problem because this subgroup includes very heterogeneous neoplasms that demand an individual analysis from the point of view of cell loss.

A separate discussion is necessary when a tumor node contains extended necrotic areas which occupy a significant part of the volume. In this case, tumor oxygenation will be highly influenced by the anoxic area characterizing a necrotic tissue. The selection of the region of interest is, in this situation, a difficult matter. The used method of manual contouring may lead to an incorrect selection of the area of interest overlapping with the necrotic area. That may drastically distort the results demonstrating reduced oxygen saturation, as compared with the real value. In addition, around the necrosis area inflammation may occur, which provokes vascular reaction and increase of the blood flow being an additional distortion factor of the data on the oxygen status of the tumor. It is exactly the case represented in the clinical example. It is possible that necrotic changes of the tumor node could be a contraindication for DOS use on the present state of the method development.

In its turn, the oxygen saturation of any tissue is directly associated with the features of its blood flow, that is, dependent on the state of the vascular bed. In our investigation, an attempt was made to assess the change of the breast tumor blood flow dependent on the effect of the neoadjuvant CT. Available literature shows that early decrease or complete disappearance of the tumor blood flow evaluated by Doppler sonography is the indicator of the efficiency of CT. On the contrary, the increase of the vascularization of the tumor during therapy may be an indicator of the tumor's progression. ${ }^{38,60,61}$ In our investigation, 3 weeks after therapy, a certain decrease of tumor vascularization was observed in the overwhelming majority of the patients. In four tumors with the first, second, and third degrees of tumor response a growth of the blood flow was detected, rather significant in two cases (Fig. 3). Those changes were observed in patients with triple-negative $\mathrm{BC}$ for which occurrence of necrotic areas and development of a peritumoral inflammation is distinguishing. We may assume that the multiple increase of the blood flow may be explained in these patients by exactly that fact. The absence of the correlation of the blood flow change and the response of the tumor may be explained as follows. The limit of resolution of contemporary ultrasonic devices is confined by blood vessels of minimum $0.1 \mathrm{~mm}$ diameter, ${ }^{62}$ changes in which do not show the processes in the capillary vessel network due to tumor's neoangiogenesis. It is evident that for the assessment of processes occurring in the tumor's vascular network methods of higher resolution are required which enable imaging of vessels with diameters of 10 to $100 \mu \mathrm{m}$.

\section{Conclusion}

Oxygen saturation level of BC under short-term chemotherapeutic impacts may allow for a prognosis of the tumor response to the therapy. Increased oxygen saturation is a predictive factor of 
the fourth and fifth degrees of pathological tumor response. A reduction or absence of the oxygen saturation dynamics allows for assumption of the first or the second degree of pathological tumor response. The most complicated is the prognosis of the third degree of pathomorphism, since in such cases both increasing and decreasing of the oxygen saturation level can be observed.

\section{Disclosures}

The authors have no relevant financial interests in this article and no potential conflicts of interest to disclose.

\section{Acknowledgments}

Projects of Russian Foundation of Basic Research \# 15-4202528 and 15-29-04884 for financial support.

\section{References}

1. J. Ferlay et al., "GLOBOCAN 2012 v1.1, cancer incidence and mortality worldwide: IARC cancerbase No. 11," International Agency for Research on Cancer, Lyon, France, https://www.wcrf.org/int/cancerfacts-figures/data-specific-cancers/breast-cancer-statistics (2014).

2. B. Fisher, "Laboratory and clinical research in breast cancer: a personal adventure. The David A. Karnofsky memorial lecture," Cancer Res. 40, 3863-3874 (1980).

3. J. Broadwater, "Mastectomy following preoperative chemotherapy. Strict operative criteria control operative morbidity," Ann. Surg. 213(2), 126-129 (1991).

4. Early Breast Cancer Trialists' Collaborative Group (EBCTCG), "Comparisons between different polychemotherapy regimens for early breast cancer: meta-analyses of long-term outcome among 100,000 women in 123 randomised trials," Lancet 379(9814), 432444 (2012).

5. J. F. Waljee, "Neoadjuvant systemic therapy and the surgical management of breast cancer," Surg. Clin. 87, 399-415 (2007).

6. H. M. Kuerer, "Integration of neoadjuvant chemotherapy and surgery in the treatment of patients with breast carcinoma," Breast Dis. 12, 69-81 (2001).

7. P. Cortazar et al., "Pathological complete response and long-term clinical benefit in breast cancer: the CTNeoBC pooled analysis," Lancet 384(9938), 164-172 (2014).

8. W. J. Gradishar et al., "Breast cancer version 3.2014," J. Natl. Comp. Cancer Network 12, 542-590 (2014).

9. G. Von Minckwitz et al., "Definition and impact of pathologic complete response on prognosis after neoadjuvant chemotherapy in various intrinsic breast cancer subtypes," J. Clin. Oncol. 30(15), 1796-1804 (2012).

10. H. Imai, "Economic evaluation of the prevention and treatment of breast cancer-present status and open issues," Breast Cancer 14(1), 81-87 (2007).

11. B. Stewart and P. Klrihus, World Health Organisation. World Cancer Report. International Agency on Research for Cancer, pp. 188-190, IARC Press, Lion (2003).

12. A. M. Gonzalez-Angulo, F. Morales-Vasquez, and G. N. Hortobagyi, "Overview of resistance to systemic therapy in patients with breast cancer," in Advances in Experimental Medicine and Biology, Vol. 608, pp. 1-22, Springer, New York (2007).

13. D. Balmativola et al., "Pathological non-response to chemotherapy in a neoadjuvant setting of breast cancer: an inter-institutional study," Breast Cancer Res. Treat. 148(3), 511-523 (2014).

14. M. Ono et al., "Tumor-infiltrating lymphocytes are correlated with response to neoadjuvant chemotherapy in triple-negative breast cancer," Breast Cancer Res. Treat. 132(3), 793-805 (2012).

15. E. Boudewijn et al., "Optical mammography using diffuse optical spectroscopy for monitoring tumor response to neoadjuvant chemotherapy in women with locally advanced breast cancer," Clin. Cancer Res. 21(3), 577-584 (2015).

16. U. Sharma et al., "Longitudinal study of the assessment by MRI and diffusion-weighted imaging of tumor response in patients with locally advanced breast cancer undergoing neoadjuvant chemotherapy," NMR Biomed. 22(1), 104-113 (2009).

17. R. Rakow-Penner, B. Daniel, and G. H. Glove, "Detecting blood oxygen level dependent (BOLD) contrast in the breast," J. Magn. Reson. Imaging 32(1), 120-129 (2010).

18. D. Angelucci et al., "Long-term outcome of neoadjuvant systemic therapy for locally advanced breast cancer in routine clinical practice," J. Cancer Res. Clin. Oncol. 139(2), 269-280 (2013).

19. C. Rousseau, A. Devillers, and C. Sagan, "Monitoring of early response to neoadjuvant chemotherapy in stage II and III breast cancer by [18F] fluorodeoxyglucose positron emission tomography," J. Clin. Oncol. 24 (34), 5366-5372 (2006).

20. A. V. Maslennikova et al., "Comparative study of tumor hypoxia by diffuse optical spectroscopy and immunohistochemistry in two tumor models," J. Biophotonics 3(12), 743-751 (2010).

21. K. Vishwanath et al., "Using optical spectroscopy to longitudinally monitor physiological changes within solid tumors," Neoplasia 11, 889-900 (2009)

22. V. V. Tuchin, Lasers and Fiber Optics in Biomedical Science, p. 384, Saratov State University Publishing, Saratov (1998).

23. D. A. Boas et al., "Imaging the body with diffuse optical tomography," IEEE Signal Process Mag. 18(6), 57-75 (2001).

24. P. Taroni et al., "Mapping of calf muscle oxygenation and haemoglobin content during dynamic plantar flexion exercise by multi-channel timeresolved near-infrared spectroscopy," Phys. Med. Biol. 49, 685-699 (2004).

25. X. Intes and C. Chance, "Non-PET functional imaging techniques: optical," Radiol. Clin. North Am. 43(1), 221-234 (2005).

26. A. G. Orlova et al., "Frequency-domain diffuse optical tomography with single source-detector pair for breast cancer detection," Laser Phys. Lett. 5(4), 321-327 (2008).

27. K. Vishwanath et al., "Using optical spectroscopy to longitudinally monitor physiological changes within solid tumors," Neoplasia 11, 889-900 (2009).

28. V. Maslennikova et al., "Non-invasive optical assessment method of the oxygen status of breast neoplasms," Tumors Feminine Reprod. Syst. 1, 5-10 (2010).

29. T. D. O'Sullivan et al., "Optical imaging correlates with magnetic resonance imaging breast density and reveals composition changes during neoadjuvant chemotherapy," Breast Cancer Res. 15, 14 (2013).

30. Q. Zhu et al., "Benign versus malignant breast masses: optical differentiation with US-guided optical imaging reconstruction," Radiology 237(1), 57-66 (2005).

31. B. J. Tromberg et al., "Non-invasive in vivo characterization of breast tumors using photon migration spectroscopy," Neoplasia 2, 26-40 (2000).

32. B. J. Tromberg et al., "Imaging in breast cancer: diffuse optics in breast cancer: detecting tumors in pre-menopausal women and monitoring neoadjuvant chemotherapy," Breast Cancer Res. 7, 279-285 (2005)

33. J. Shudong et al., "Predicting breast tumor response to neoadjuvant chemotherapy with diffuse optical spectroscopic tomography prior to treatment," Clin. Cancer Res. 20(23), 6006-6015 (2014).

34. M. van de Giessen, B. E. Schaafsma, and A. Charehbili, "Early identification of non-responding locally advanced breast tumors receiving neoadjuvant chemotherapy," Proc. SPIE 9303, 93032K (2015).

35. P. G. Anderson et al., "Optical mammography in patients with breast cancer undergoing neoadjuvant chemotherapy," Acad. Radiol. 24(10), 1240-1255 (2017).

36. P. G. Anderson et al., "Individual response to neoadjuvant chemotherapy assessed with optical mammography in patients with breast cancer," Proc. SPIE 10059, 100590L (2017).

37. A. E. Cerussi et al., "Diffuse optical spectroscopic imaging correlates with final pathological response in breast cancer neoadjuvant chemotherapy," Philos. Trans. A Math. Phys. Eng. Sci. 369(1955), 45124530 (2011).

38. C. Balu-Maestro et al., "Evaluation de la réponse tumorale à la chimiothérapie par l'IRM et l'écho Doppler couleur,' Le Sein 6, 194-202 (1996).

39. M. Toi et al., "Visualization of tumor-related blood vessels in human breast by photoacoustic imaging system with a hemispherical detector array," Sci. Rep. 7, 41970 (2017). 
40. C. Lal and M. Leahy, "An updated review of methods and advancement in microvascular blood flow imaging," Microcirculation 23(5), 345-363 (2016).

41. Y. Shang, T. Li, and G. Yu, "Clinical applications of near-infrared diffuse correlation spectroscopy and tomography for tissue blood flow monitoring and imaging," Physiol. Meas. 38(4), R1-R26 (2017).

42. T. Durduran and A. G. Yodh, "Diffuse correlation spectroscopy for non-invasive, micro-vascular cerebral blood flow measurement," NeuroImage 85, 51-63 (2014).

43. E. M. Buckley et al., "Diffuse correlation spectroscopy for measurement of cerebral blood flow: future prospects," Neurophotonics 1, 011009 (2014).

44. C.-G. Bangalore-Yogananda et al., "Concurrent measurement of skeletal muscle blood flow during exercise with diffuse correlation spectroscopy and Doppler ultrasound," Biomed. Opt. Express 9(1), 131-141 (2018).

45. L. Dong et al., "Diffuse optical measurements of head neck tumor hemodynamics for early prediction of chemoradiation therapy outcomes," J. Biomed. Opt. 21(8), 085004 (2016).

46. Q. Zhu, S. H. Kurtzman, and P. Hegde, "Utilizing optical tomography with ultrasound localization to image heterogeneous hemoglobin distribution in large breast cancers," Neoplasia 7(3), 263-270 (2005).

47. W. H. Kuo et al., "Vascularity change and tumor response to neoadjuvant chemotherapy for advanced breast cancer," Ultrasound Med. Biol. 34, 857-866 (2008).

48. A. Kumar et al., "Color Doppler ultrasonography for treatment response prediction and evaluation in breast cancer," Future Oncol. 6(8), 12651278 (2010).

49. G. Singh et al., "Role of color Doppler indices in predicting disease-free survival of breast cancer patients during neoadjuvant chemotherapy," Eur. J. Radiol. 75, e158-e162 (2010).

50. K. N. Ogston et al., "A new histological grading system to assess response of breast cancers to primary chemotherapy: prognostic significance and survival," Breast 12(5), 320-327 (2003).

51. B. J. Tromberg et al., "Predicting responses to neoadjuvant chemotherapy in breast cancer: ACRIN 6691 trial of diffuse optical spectroscopic," Cancer Res. 76(20), 5933-5944 (2016).

52. M. V. Pavlov et al., "The complex evaluation of tumor oxygen state and vasculature during preoperative chemotherapy in patients with breast cancer," Proc. SPIE 10059, 100590P (2017).

53. H. Lu et al., "Sustained poststimulus elevation in cerebral oxygen utilization after vascular recovery," J. Cereb. Blood Flow Metab. 24(7), 764-770 (2004).

54. B. M. Fenton, E. M. Lord, and S. F. Paoni, "Intravascular $\mathrm{HbO}_{2}$ saturations, perfusion and hypoxia in spontaneous and transplanted tumor models," Int. J. Cancer 93, 693-698 (2001).

55. J. M. Brown and A. J. Giaccia, "The unique physiology of solid tumors: opportunities (and problems) for cancer therapy," Cancer Res. 58, 1408-1416 (1998).

56. G. Powathil et al., "Modeling the spatial distribution of chronic tumor hypoxia: implications for experimental and clinical studies," Comput. Math. Methods Med. 2012, 410602 (2012).

57. D. J. Brenner et al., "A convenient extension of the linear-quadratic model to include redistribution and reoxygenation," Int. J. Radiat. Oncol. Biol. Phys. 32, 379-390 (1995).

58. J. A. Bertout, S. A. Patel, and M. C. Simon, "The impact of $\mathrm{O}_{2}$ availability on human cancer," Nat. Rev. Cancer 8, 967-975 (2008).

59. Q. Zhu et al., "Pathologic response prediction to neoadjuvant chemotherapy utilizing pretreatment near-infrared imaging parameters and tumor pathologic criteria," Breast Cancer Res. 16(5), 456 (2014).

60. J. S. Walsh et al., "Color Doppler studies of axillary node metastases in breast carcinoma," Clin. Radiol. 49, 189-191 (1994).

61. W. T. Yang, J. Chang, and C. Metreweli, "Patients with breast cancer: difference in color Doppler flow and gray-scale US features of benign and malignant axillary lymph nodes," Radiology 215, 568-573 (2000).

62. R. J. Schroeder, "Role of power Doppler techniques and ultrasound contrast enhancement in the differential diagnosis of focal breast lesions," Eur. Radiol. 13, 68-79 (2003).

Mikhail Viktorovich Pavlov is a doctor of ultrasound diagnostics at the Volga Region Medical Center. He graduated from the Nizhny Novgorod State Medical Academy in 2011. His major research area focuses on imaging for breast cancer diagnostics and monitoring of antitumoral treatment. His interests include the investigation of the capabilities of diffuse optical spectroscopy (DOS) and multimodal ultrasound in the evaluation of the effectiveness of preoperative chemotherapy in patients with breast cancer.

Tatyana Igorevna Kalganova is a senior researcher in the Laboratory of Regenerative Medicine in IBT NNSMA. She received her $\mathrm{PhD}$ in biology in 2013 from Voronezh State University. Her major research area focuses on optical imaging for cancer diagnostics and investigation. Her interests include the investigation of capabilities of DOS in tumor hypoxia detection and monitoring. She is also interested in noninvasive monitoring of tumor oxygen levels under different conditions.

Yekaterina Sergeevna Lyubimtseva graduated from Nizhny Novgorod State Medical Academy in 2007. Since 1983 to the present time was working as oncologist-chemotherapeutist of Regional Oncological Hospital, Nizhny Novgorod, Russia. From 2017, she headed the Chemotherapy Department of Nizhny Novgorod Regional Oncological Hospital. Her major area interests include individualised chemotherapy and target treatment of breast cancer, gastrointestinal cancer, and sarcoma.

Vladimir Ivanovich Plekhanov is a leading technologist at the Institute of Applied Physics RAS, where he develops and introduces the experimental optical solutions for biological/medical applications, with experience in biomedical imaging include fluorescence imaging by photosensitizers and nanoparticals, computer vision, laser/LED irradiators, PDT include light-based therapeutic and, increasingly, TCSPC molecular-scale fluorescent decay measurement/imaging at a picoseconds time range.

German Yurievich Golubyatnikov graduated in 1983 and received his master's degree from Gorky State University. He received his PhD in plasma physics in 1996 from the Institute of Applied Physics RAS. Since 1983 to the present time, he has been with the IAP RAS, currently as a senior research fellow. His recent research interest and results are in the new technique of submillimeter microwave spectroscopy and spectroscopy of biosystems.

Olga Yevgenyevna llyinskaya graduated from Nizhny Novgorod State Medical Academy in 1993. Since 1997 to the present time, she was working as a pathologist at the Regional Oncological Hospital, Nizhny Novgorod, Russia. From 2011, she headed the Pathoanatomical Department of Nizhny Novgorod Regional Oncological Hospital. Her main areas of her interests include histological and immunohistochemical examination of various malignant tumors, pathomorphological evaluation of the effectiveness of various methods of antitumor treatment.

Anna Gennadjevna Orlova is a senior scientist at the Laboratory of Biophotonics of the Institute of Applied Physics RAS. She received her $\mathrm{PhD}$ in biology in 2004 from Nizhny Novgorod State University. Her major research area focuses on optical imaging both in clinical and experimental oncology. Her main interests include DOS in cancer detection as well as investigation of individual metabolic feature of tumors. She is the coauthor of more than 30 peer-reviewed articles and several book chapters.

Pavel Vladimirovich Subochev is the optoacoustic group leader at the Laboratory of Biophotonics, Institute of Applied Physics, RAS. He graduated from Lobachevsky State University of Nizhni Novgorod in 2006 and received his $\mathrm{PhD}$ in passive acoustic radiometry from the Institute of Applied Physics of Russian Academy of Sciences. Since 2012, he is focused on the development of new instrumentation for optoacoustic imaging of biotissues. He is a coauthor of 30 peer-reviewed articles and conference proceedings related to optoacostics.

Dmitriy Vladimirovich Safonov is a doctor of medical sciences, professor of the Radiation Diagnostics Department of Nizhny Novgorod State Medical Academy. His doctoral thesis on "Syndrome Ultrasonic Diagnosis of Lung Disease" defended in 2003, the academic title of professor awarded in 2012. His scientific directions are ultrasound diagnosis of lung and pleural diseases, shear wave elastography in the diagnosis of focal, and diffuse liver diseases. He is an author 
of 180 scientific publications, including six monographs and nine patents.

Natalia Mikhailovna Shakhova is a leading scientist at the Laboratory of Biophotonics of the Institute of Applied Physics, RAS. She received her doctor of medicine degree in 2005. Her main interests include translational medicine, oncogynecology, biophotonics, optical bioimaging, laser surgery, photodynamic diagnosing, and photodynamic therapy. She is an author of 156 scientific publications, including 20 peer-reviewed articles, 5 book chapters, 1 handbook, and 39 abstracts in conference proceedings.
Anna Vladimirovna Maslennikova is a professor at the Department of Oncology of Nizhny Novgorod State Medical Academy and a professor at the Department of Biophysics of Lobachevsky University, Nizhny Novgorod, Russia. She received her DSc degree in 2008 in radiation oncology for the thesis "Thermoradiotherapy and chemotherapy of locally advanced larynx and pharynx cancer." Her recent research interests are connected with the problem of tumor hypoxia imaging, monitoring, and correction in clinical and experimental settings. 